\title{
Altered cytoskeletal structures in transformed cells exhibiting obviously metastatic capabilities
}

LIN Zhongxiang,HAN Yaling, WU Bingquan*, FANG Weigang* Department of Cell Biology, Beijing Institute for Cancer Research * Departmont of Pathology, Beijing Medical University

\begin{abstract}
Cytoskeletal changes in transformed cells (LM-51) exhibiting obviously metastatic capabilities were investigated by utilization of double-fluorescent labelling through combinations of: (1) tubulin indirect immunofluoreseonce plus Rhodamine-phalloidin staining of F-actins; (2) indirect immunofluorescent staining with a -actinin polyclonal- and vinculin monoclonal antibodies. The LM-51 cells which showed metastatic index of $>50 \%$ were derived from lung metastasis in nude mice after subcutaneous inoculation of human highly metastatic tumor DNA transfected NIH3T3 cell transformants. The parent NIH3T3 cells exhibited well-organized microtubules, prominent stress fibers and adhesion plaques while their transformants showed remarkable eytoskeletal alterations: (1) reduced microtubules but increased MTOC fluorescence; (2) disrupted stress fibers and fewer adheaion plaques with their protein components redistributed in the cytoplasm; (3) Factin- and a -actinin/vinculin aggregates appeared in the cytoplasm. These aggregates were dot-like, varied in size (0.1$0.4 \mu \mathrm{m}$ ) and number, located near the ventral surface of the cells. TPA-induced actin/vineulin bodies were studied too. Indications that aotin and a-actinin/vinoulin redistribution might be important alterations involved in the expression of metastatic capabilities of LM-51 transformed cells were discussed.
\end{abstract}

Key words: Microtubules stress fibers, adhesion plaques, actin and a -actinin / vinculin aggregates.

\section{INTRODUCTION}

Alterations of cytoskeletal structures including reduced microtubules, disruption of stress fibers and redistribution of actin-filaments in transformed cells have been

Correspondence: LIN Zhongxiang M. D., Department of Cell Biology, Beijing Institute for Cancer Research, DaHong-Luo-Chang St., West District, Beijing 100034, China. 
Altered cytoskeletal structures in transformed cells

documented and reviewed [1-3]. However, with a few exceptions, little work has been done on what the role these alterations may play in relation to the expression of tumor cell malignancy.

Experimental and clinical data indicate that autonomous Proliferating and invasive spreading are main characters of cell malignancy. It is still largely unclear which of the genetic elements as well as the molecular and cellular events are determinants in cell malignancy and the expression of cell metastatic potential.

Miorotubules, stress fibers and membrane-associated oytoskeleton are known to be responsible for maintainanee of cell morphology[1], direction of cell locomotion[4], cell spreading, cell migration and stability of cell-substrate contact[5] in normal cells. Accordingly, it has been suggested that the changes of these oytoskeletons in transformed cells may be involved in oytodynamic processes related to tremor cell dissemination and implantation which are based on cell proliferating-and metastatic capabilities [6-8]. Therefore, it is intriguing to investigate eytoskeletal changes in tumor cells with special respect to their metastatic capabilities.

Motivated by the cytosketetal changes induced by viral transformation [9, 10] and by the indications that the oytoskeleton may be the target site for oncogene products[11], we have investigated the distribution of tubulin, F-actin, a-actinin and vinculin in human tumor DNA transformed cells exhibiting obviously metastatic capabilities. This transformed cell line LM-51 was derived from the lung metastasis of nude mice after subcutaneous inoculation of transformed cell clone which was obtained from the secondary transformants of NIH3T3 cells transfected with human highly metastatic tumor cell DNA [12]. We have demonstrated human Alu sequences in DNA genome of the transformants, thus to confirm that the transformants were incorporated with human DNA fragments. The LM-51 cells exhibit metastatic index of $>50 \%$ in nude mice after subcutaneous inoculation. On the contrary, the parent NIH3T3 cells have been demonstrated to be non-metastatic under the same condition. The non-metastatic parent NIH3T3 cells showed well organized mierotubules, prominent stress fibers and large, well developed vinculin adhesion plaques while their transformants, the LM-51 cells showed: (1) reduced microtubules but increased mierotubule organizing center (MTOC)fluorescence; (2) disrupted stress fibers and reduced adhesion plaques with their protein components redistributed in the cytoplasm; (3) many F-actin- and a-actinin/ vinculin aggregates appeared in the cytoplasm. These aggregates, varying in size $(0.1-$ $0.4 \mu \mathrm{m}$ ) and number, were located near the ventral surface of the cell. We have also seen TPA-induced actin/vinculin bodies in myocytes. The common topological arrangement of a-actinin/vinculin and F-actin in both circumstances is discussed. It is suggested that the redistribution of actin and a-actinin/vinculin might be important alterations involved in the expression of metastatic capabilities of LM-51 cells.

\section{MATERIALS AND METHODS}

\section{Cells}

NIH3T3 cell line was a gift from Department of Pathology of NCI, NIH, USA. LM-51 transformed cell line was derived from lung metastasis of nude nice which were, 
subcutaneously inoculated with human highly mteastatic tumor DNA transfected NIH3T3 cell transformants [12]. Cells were grown in Dulbecco's MEM containing 15\% calf serum in an atmosphere of $5 \% \mathrm{CO}_{2}$ and $95 \%$ air at $37^{\circ} \mathrm{C}$. Primary cultures of day- 12 chicken embryonic skeletal muscle cells were grown in Eagle's MEM containing 10\% horse serum, $10 \%$ chicken embryonic extract in the same condition discribed above. The seeded mononucleated myoblasts started fusion, formed myotubes in day-2 cultures, and grew gradually with increased number and size; striated myotubes showing spontaneous contractioncould be observed in day-4 cultures [13].

\section{Antibodies}

Tubulin antibodies were purchased from Miles Scientific Co. (Elkhart, IN). The mouse monoclonal antibody against chicken gizzard vinculin, VIN-II-5 (ION Immunobiological, Lisle) reacts specifically with vinculin on immunoblots and stains adhesion plaques of cultured cells briefly fixed in formalin. Guinea pig antibody against smooth muscle a -actinin (a gift of Dr. Hans-Peter Kapprell, University of Cologne) stains the dense bodies of stress fibers in nonmuscle cells in a punctate fashion as well as lightly stained Z-bands and their precursor structures in myogenic cells. FITC-or Rhodamine conjugated goat antibodies against rabbit-, mouse-or guinea pig IgG were purchased from Miles Scientific Co.

\section{Immunofluorescence- and electron-microscopy}

Cells were processed for immunofluorescence microscopy as following [13]: Cultures were rinsed with PBS and fixed for $3 \mathrm{~min}$ in $2 \%$ formaldehyde (freshly prepareh from varaformaldehyde) in PBS. The cells were then permeabilized and soluble proteins were extracted with $0.5 \%$ Triton X-100 in PBS. This PBS-Triton solution was also used for all subsequent antibody washing steps.

Double-fluorescent labelling method: Cells were fixed as described above; then were incubated with guinea pig anti- $\alpha$-actinin antibody and mouse momoclonal antibody against vinculin in appropriate dilutions. After the primary antibody incubation, cells were washed three times, incubated with the two secondary antibodies, goat anti-guinea pig IgG(Rhodamine conjugated) and goat anti-mouse IgG (FITC conjugated) simultaneously, and then were washed again. For double labelling of tubulin and F-actins, cells were rinsed wlth MES buffer [(2 N-Moapholino)-ethanesulfonic acid, Sigma Co.] (0.1M MES; $1 \mathrm{mM} \mathrm{MgCl} 2 ; 1 \mathrm{mM}$ EGTA; 4\% $\mathrm{PEG}_{8000}$. pH6.75), extracted in $0.2 \%$ Triton X-100 in MES buffer for 2 rain; rinsed in MES buffer, and fixed with $2 \%$ glutaraldehyde in MES buffer for $15 \mathrm{~min}$ at room temperature. After fixation, cells were rinsed in PBS three times, then treated with $\mathrm{NaBH}_{4}(1 \mathrm{mg} / \mathrm{ml}$ in PBS) for $4 \mathrm{~min}$, three times; and rinsed in PBS before incubation with the primary antibody. Cells were then washed and incubated with FITCconjugated goat anti-rabbit IgG and washed again. Cells were then stained with Rhodamine-phalloidin(Moleoular Probes Co. USA.) which binds specifically to F-actins to visualize stress fibers, actin filaments and thin flamemts in nonmusele cells and muscle cells.

Cells were examined with an Opton epifluoneseence microscope using excitation filters for either FITC or Rhodamine fluorescence, and a short-band pass barrier filter for fluoresoein that eliminates most bleed-through between channels. ASA 400 black and white 
Altered cytoskeletal structures in transformed cells

film (Lucky, China) was used.

The procedures for electron microscopy were those described previously [14].

\section{Tumor transplantation and metastasis experiments in nude mice}

Balb/cA nu/nu nude mice (from the Division of Laboratory Animal Science, Beijing Medical University), 8-13 weeks old, were grown in standard conditions described previously[15]. LM-51 cell suspension $\left(1-2 \times 10^{6}\right.$ cells in $0.5 \mathrm{ml}$ culture medium) was inoculated subcutaneously on the back of nude mice. Tumor nodules were formed in 2 weeks and metastatic fool in regional and/or distant lymph node and lung tissue were observed after 5 weeks in sacrificed or spontaneously died mice and were judged histologically.

\section{Treatment of chicken embryonic skeletal muscle cells with TPA}

Day-4 muscle cultures were exposed to TPA (Sigma Co. $75 \mathrm{ng} / \mathrm{ml}$ ) for periods of 1 to $72 \mathrm{hrs}$ and were fixed in different time courses.

\section{RESULTS}

\section{Establishment of LM-51 transformed cell line and its metastatic properties}

NIH3T3 cells were transfected with DNA fragments from a highly metastatic human lung cancer cell line. The tumor DNA-induced transformants were obtained and examined as reported previously[12]. Metastasis of the TF-87-2-10 cell clone, one of the secondary transformants, was proved in nude mice. The tumors at th9 primary inoculation site and metastasis were of fibrosarcoma cell type as judged histologically.

Gultured LM-51 cell line was derived from the lung metastasis of the secondary transformant TF-87-2-10 cell clone after subcutaneous inoculation into nude mice. The LM-51 cells are thick, fusiform in shape, with a tendency to form multilayers and fool in culture. They grow fast, with the generation time of $17 \mathrm{hr}$. These cells were low adhesive and easily to detach from the substrate. In nude mice transplantation experiments, LM-51 cells are $100 \%$ tumorigenic and obviously metastatic, with a metastatic index of $>50 \%$.

The parent NIH3T3 cells were inoculated into the back of nude mice as control group. Observations were made in parallel with yhe transformed cell groups. Judged with the criteria used for the transformed cell groups, the parent NIH3T3 cells have been proved to be non-metastatic.

\section{Tubulin immunofluorescence}

\section{NIH3T3 cells}

Elaborate cytoplasmic miorotubules were shown. They radiated out from the MTOC at the perinuclear area and reached the cell border. Noteworthily: (1) Fine, long microtubules could be followed to their ends at the cytoplasmic process near the ruffled membrane, the latter was decorated with Rho-phalloidin staining (Fig. la, lb, broad arrows). (2) In the same cell, microtubules were arranged in a more or less parallel 
pattern in the spreading area of the cytoplasm, but were less developed in the retracbed area of the cytoplasm.

\section{LM-51 transformed cells}

Diminished mierotubules were observed. The MTOC fluorescence consisting of the central diffused tubulin and a few tubulin strands was conspicuous (Fig.1c,1e). More than $90 \%$ of the cells could be recognized in this type. Only less than $10 \%$ cells displayed microtubules which were still fewer than in parent NIH3T3 cells.

\section{Rho-pkalloidin staining and a-actinin immunofluorescence NIH3T3 cells}

(1) Rhodamine-phalloidin stained prominent stress fibers were observed. They were straight and parallel to the longitudinal axis of the cells (Fig. 1b, thin arrow). Rho-phalloidin stained polygonal geodesic dome-like networks[16] were also seen in the spreading area of the cytoplasm (Fig. 1b). From the vertices of the networks, stress fibers stretched out toward the Rho-phalloidin stained ruffled membrane (Fig. 1b, broad arrow). (2) a actinin immunofiuoreseent staining revealed stress fibers in a punctate fashion with intense fluorescence at the termini (the adhesion plaques) of the stress fibers (Fig. 2a).

\section{LM-51 transformed cells}

Decreased stress fibers were observed. (1) Rho-phalloidin staining revealed that actin aggregates were distributed in the vicinity of the nucleus. These aggregates were dot-like, varied in size and number; some of them were arranged in short strands along the surviving stress fibers that looked like broken or poorly assembled actin structures (Fig. 1d, 1f). (2) a -actinin immunofluorescence also showed disruption of stress fibers and appearance of the a -actinin aggregates which were somehow comparable to the F-actin aggregates in their size $(0.1-0.4 \mu \mathrm{m})$, number and location. Some short, lined-up a actinin aggregates were also seen though most of the a-actinin positive punctate stress fibers were exclusively disappeared (Fig. 2c).

\section{Vinculin fluorescence \\ NIHST8 cells}

The vinculin antibody stained adhesion plaques were large, well-organized and distributed at the ends of stress fibers. Double fluorescent staining showed co-localization of vinculin with a -actinin at adhesion plaques (Fig. 2a, 2b). By changing the focus plane, it was observed that these adhesion plaques were located near the ventral surface of the cell.

\section{LM-51 transformed cells}

The vinculin adhesion plaques were disrupted and reduced in number and size. Simultaneously, vinculin positive aggragates appeared in the cytoplasm; they were colocalized with the a -actinin aggregates (Fig. 20,2d). The topological continuity between vinculin aggregates and the surviving adhesion plaques was observed. It may imply the cytodynamic process involving the reorganization of vinculin molecules from adhesion 
Altered cytoskeletal structures in transformed cells

plaques to the assembly of aggregates.

\section{Tumor promoter TPA-induced actin/vinculin bodies in myotubes-lmmuno- fluorescence and EM analysis}

Exposure of day-4 cultures of chicken embryonic skeletal muscle cells to TPA (75 ng/ $\mathrm{ml}$ ) for $5 \mathrm{hr}$ led to the disruption of myofibrillar I-Z-I complexes and reorganization of a-actin into small $(3 \mu \mathrm{m})$ bodies, which were colocalizod with vinculin rims (Fig. $3 \mathrm{~b}$, $3 \mathrm{e}$ ). Those bodies were located near the cortical cytoplasm and wore named GAB (cortica2 actin bodies) [14]. Electron microscopy has confirmed the immunocytochemical observation of the CABs. Sections through CABs (Fig. 3d) in 5--10 hr TPA-treated myotubes revealed that those CABs displayed a central, tight filamentous network which, following decoration with heavy meromyosin, form typical arrowheads [14]. Coated vesicles were often seen in the periphery. They are not bounded by membrane. IFs and thick filaments are excluded.

\section{DISCUSSION}

For the purpose of investigating the metastatic processes at the molecular and cellular level, it has been crucial to compare the transformed cells that express positive metastatic capabilities with their non-metastatic counterpart cells. In this respect, LM-51 cells are suitable experimental matorial since: (i) they exhibit a metastatic index of $>50 \%$. in nude mice; (2) their motastatic behaviours may involve the cytodynamio process of cell release from the original nodules at subcutaneous site, through circulation, to form distant lung metastases; (3) evidence that human DNA sequences have been incorporated into the DNA genome of the transformants is indicative of control at DNA level for the expression of metastatic activities [12]; (4) they have their non-metastatic parent NIH3T3 cells as counterpart. The rapid proliferation activity, thick fusiform shape and the detaching tendency of LM-51 cells in culture are also agreeable with and characteristic of the metastatic potential of these cells.

Studies using high- and low metastatic variants of the same tumor cell lines suggested that a large number of factors, including extrinsic factors such as those from the host, and intrinsic factors which depend predominatly on the activity of the individual tumor cell may be involved in the spread of neoplastic cells throughout the body. Among those intrinsic factors which may affect the metastatic potential, it seems that cell motility and capacity of cells to break existing cell-cell and cell-substrate contacts are the most probable ones $[7,8]$. The fact that treatment with cytoskeleton-disrupting drugs induces marked alterations in the metastatic properties of tumor cells emphasizes the possible involvement of cytoskeletons in metastatic process $[17,18]$.

The above rationale and suggestions are supported by results obtained in our work. The findings that two of three major eytoskeletal networks including microtubules, microfilaments and cell-substrate contact systems were altered dramatically in LM-51 cells strongly suggest the involvement of the eytoskeletons in the expression of cell malignancy, especially the metastatic properties.

It has been suggested that depolymerization of microtubules in early cell cycle is sufficient to initiate DNA synthesis $[19,20]$. Accordingly, it is possible that depolymeriza- 
tion of microtubules in transformed cells may correlate to their active DNA replication and cell proliferation [6]. Furthermore, since microtubules may play essential roles in direction of cell locomotion and in the stabilization of nascent cell-substrate contacts in normal cell migration[4], it is likely that the depolymerization of microbubules might be relevant to the disordered, actively motile properties, the detaching tendency and the metastatic potential of LM-51 cells.

Many studies in recent years have demostrated the association between the transformation of cells by oncogeneo viruses and the dramatic alterations of the microfilament system $[2,5,9,10,21]$. The observed changes of stress fibers as well as vinculin adhesion plaques in LM-51 cells were similar to those described in such viral transformed cells and in the highly metastatic variants of mouse melanoma and fibrosarcoma $[7,8]$. The disassembled stress fibers, reduced vinculin adhesion plaques and redistribution/ reorganization of their protein components into aggregates have been correlated to the active motility and invasive properties, hence the metastatic potential of the transformed cells $[7,8,21]$. This correlation was further supported by the evidence [22--24] that colocalization of cell surface plasminogen activator urokinase and protease degradation activities with vinculin in the redistributed topological pattern in cultured tumor cells and transformed cells resembled the aggregates previously reported in virus transformed cells $[9,10,21]$. Thus, it is possible that the loss of stable cellular contacts and the disarray of actin bundles often attributed to transformed cells are not strictly related to the transformation per se but may reflect the malignant metastatic potential of the transformed cells[8].

Our results are in support to the notion [10] that characteristic actin patches or aggregates occur in a variety of transformed cells irrespective of the cause of transformation. Although presently supported only by a narrow range of transformed cells, patch/ aggregate formation may reflect a common trait of transformed cells. The presented evidence, though far from enough, will nevertheless provide clues to the mechanisms underlying the phenotypio changes and the metastatic potential in malignant cells.

At present, we do not know what cellular events may control the observed alterations of oytoskeletons in transformed cells. However, it has been demonstrated recently [25] that the reorganized F-actin aggregates in virally transformed cells are highly dynamic structures. To further the invesMgation of the control of molecular assembly/disassembly of protein isoforms, TPA-induced reorganization of contractile protein isoforms in nonmuscle cells[26, 27] and muscle cells[13, 14] would be useful. We have found TPA-induced actin/vinculin bodies in myotubes. The common topological arrangement of protein isoforms may suggest a kind of molecular sorting out and subsequent self-assembly [14]. This cell-model may shed lights to the further understanding of regulation mechanism of the expression of cell malignancy.

\section{ACKNO WLEDGEMENT}

(1) The authors want to thank Mr. J. Dennis (Department of (Sell Biology, School of Medicine, Cornell University, New York, USA) for his help in EM work. (2) Part of this work was done in collaboration with Dr. H. Holtzer (Departament of Anatomy, School of Medicine, University of Pennsylvania, Philadelphia, PA, USA) 


\section{Altered cytoskeletal structures in transformed cells}

\section{REFERENCES}

[ 1 ] Brinkley BR, Fistel SH, Marcum JM, Pardue RL. Microtubules in cultured cells; Indirect immunofluoresent staining with tubulin antibody. Intl Bey Cytol 1980; 63: 59-95.

[ 2 ] Ben-Ze'ev A, The cytoskeleton in cancer cells. Biochim Biophysica Acta 1985; 780: 197-212.

[ 3 ] Lin ZX, Wang DS, Lei S J, Wang KR. Studies on microtubules of normal and tumor cells in culture: II. Observations on intracellular microtubules of cultured tumor cells by indirect immunofluorescence microscopy. Acta Biologiae Exporimentalis Sinica 1984; 17: 377-389.

[ 4 ] Rinnerthaler G, Geiger B, Small JV. Contact formation during fibroblast locomotion: Involvement of membrane ruffles and microtubules. J Cell Biol 1988; 106: 747-760.

[ 5 ] Burridge K. Substrate adhesions in normal and transformed fibroblasts: Organization and regulation of cyteskeletal, membrane and extracellular matrix components at focal contacts. Cancer Rev 1986; 4: 18-78.

[ 6 ] Kamech N, Seif R. Effect of microtubule disorganizing or overstabilizing drugs on the proliferation of rat 3T3 cells and their vitally induced transformed derivatives. Cancer Res 1988; 48: 4892-4896.

[ 7 ] Volk T, Geiger B, Raz A. Motility and adhesive properties of high-and low-metastatic routine neoplastic cells. Cancer Res 1984; 44: 811-824.

[ 8 ] Raz A, Geiger B. Altered organization of cell-substrate contacts and membrane-associated cytoskeleton in tumor cell variants exhibiting different metastatic capabilities. Cancer Res 1982; 42: 5183-5190.

[ 9 ] David-Pfeuty T, Singer SJ. Altered distributions of the cytoskeletal proteins vinculin and a -actinin in cultured fibreblasts transformed by Rous sarcoma virus. Proc Natl Acad Sci USA 1980; 77: 6687-6691.

[10] Carley WW, Barak LS, Webb WW. F-actin aggregates in transformed cells. J Cell Biol 1981; 90: 797-802.

[11] Rohrschneider LR, Rosok M J, Gentry LE. Molecular interaction of the src gene product with cellular adhesion plaques. Progr Nucleic Acid Res and Molecular Biol. 1983; 29: 233-244.

[12] Zeng GC, Fang WG, Wu BQ. Metastatic characteristic of DNAs from a highly metastatic human lung cancer cell line. Chinese Biochemical J 1988; 4: 184-189.

[13] Lin ZX, Eshelman JR, Forry-Schaudies S, Duran S, Lessard JL, Holtzer H. Sequential disassembly of myofibrils induced by myristate acetate in cultured myotubes. J Cell Biol 1987; 105:1365-1376.

[14] Lin ZX, et al. Differential response of myofibrillar and cytoskeletal proteins in cells treated with phorbol myristate acetate. J Cell Biol 1989; 108: 1079-1091.

[15] Wu BQ, et al. Establishment of highly metastatic human tumor cell lines in nude mice. National Cancer J China 1985; 7: 324-328.

[16] Lazarides E. Actin, a -actinin, and tropomyosin interaction in structural organization of actin filamentsin nonmuscle cells. J Cell Biol 1976; 68: 202-219.

[17] Hart IR, Raz A, Fidler IJ. Effect of cyteskeleton disrupting agents on the metastatic behaviour of melanoma cells. J Natl Cancer Inst 1980; 64: 891-900.

[18] Mareel M, De Brabander M. Effect of microtubule inhibitor on malignant invasion in vitro. J Natl Cancer Inst. 1978; 61: 787-792.

[19] Crossin KL, Carney DH. Evidence that microtubule depolymerizatlon early in the cell cycle is sufficient to initiate DNA synthesis. Cell 1981; 23: 61-71.

[20] Grossin KL, Carney DH. Microtubule stabilizatlon by taxol inhibits initlation of DNA synthesis by thrombin and by epidermal growth factor. Cell 1981; 27: 341-350.

[21] Marchisio PC, Cirillo D, Teti A, Zambonin-Zallone A, Tarone G. Rous sarcoma virns-transformed fibroblasts and cells of monocyte origin display a peculiar dot-like organization of cytoskeletal proteins involved in microfilament-membrane interactions. Exp Cell Res 1987; 169: 202-214.

[22] Hebert CA, Baker JB, Linkage of extracellular plasminogeu activator to the fibroblast cytoskeleton: Colocalization of cell surface urokinase with vinculin. J Cell Biol 1988: 106. 1241-1247.

[23] Chen WT, Wang J, Hasegawa T, Yameda KM. Regulation of fibronectin receptor distribution by transformation, exogenous fibronectin, and synthetic peptides. J Cell Biol 1986; 103: 1649-1661.

[24] Chen WT, Olden K, Bernard BA, Chu FF. Expression of transformation-associated protease(s) that degrade fibronectin at cell contact sites. J Cell Biol 1984; 98: 1546-1555.

[25] Stickel SK, Wang YL, Alpha-actinin-containing aggregates in transformed cells are highly dynamic structures. J Cell Biol 1987; 104:1521-1526.

[26] Schliwa M, Nakamura T, Porter KR, Eutenener U. A tumor promoter induces rapid and coordinated reorganization of actin and vinculin in cultured cells. J Cell Biol 1984; 99: 1045-1059.

[27] Meigs JB, Wang YL. Reorganization of alpha-actinin and vinculin induced by a phorbol ester in living cells. J Cell Biol 1986; 102:1430-1438.

Received 25-4-1989. Revised 2-12-1989. Accepted 16-3-1990. 
Lin ZX et al.

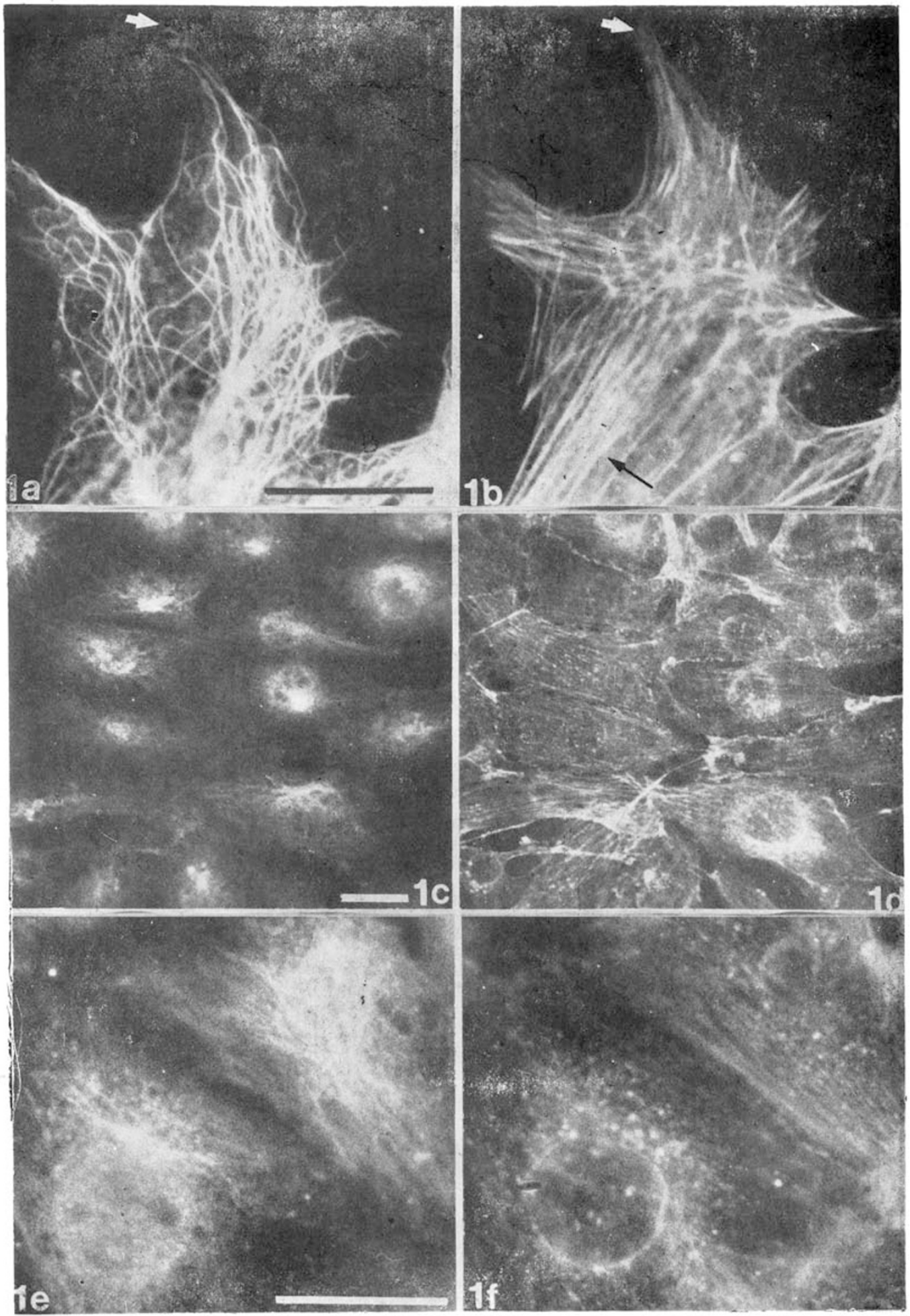

Fig. 1 Double-labelling of tubulin immunofluorescence (a, c, e) and Rho-phalloidin stained actin fluorescence (b, d, f) in a NIH3T3 cell (a \& b, the same field) and LM-51 transformed cells (c \& d; e \& f, from the same field respectively). Broad arrow in (b) pointing to actin positive ruffling membrane of a cytoplasmic process. Thin arrow in (b) pointing to parallel arrays of stress fibers, bar: $10 \mu \mathrm{m}$ 
Altered cytoskeletal structures in transformed cells
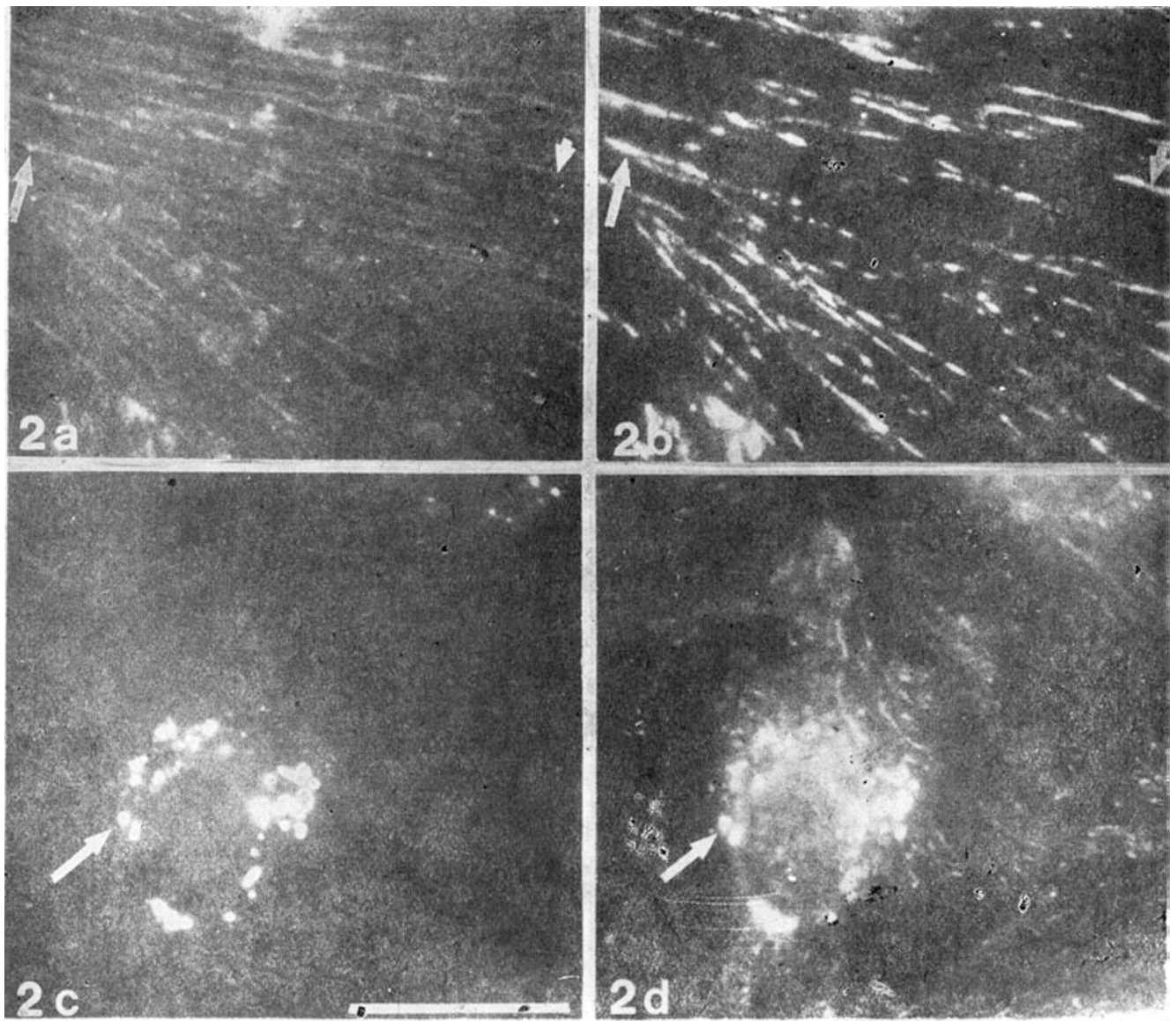

Fig. 2 Double-labelling of a-actinin ( $a, c)$ and viuculin (b, d) immunofluorescence in a NIH3T3 cell(a, b, same field) and a LM-51 transformed cell (c, d, same field). Arrows are for reference, bar: $10 \mu \mathrm{m}$. 
Lin ZX et al.
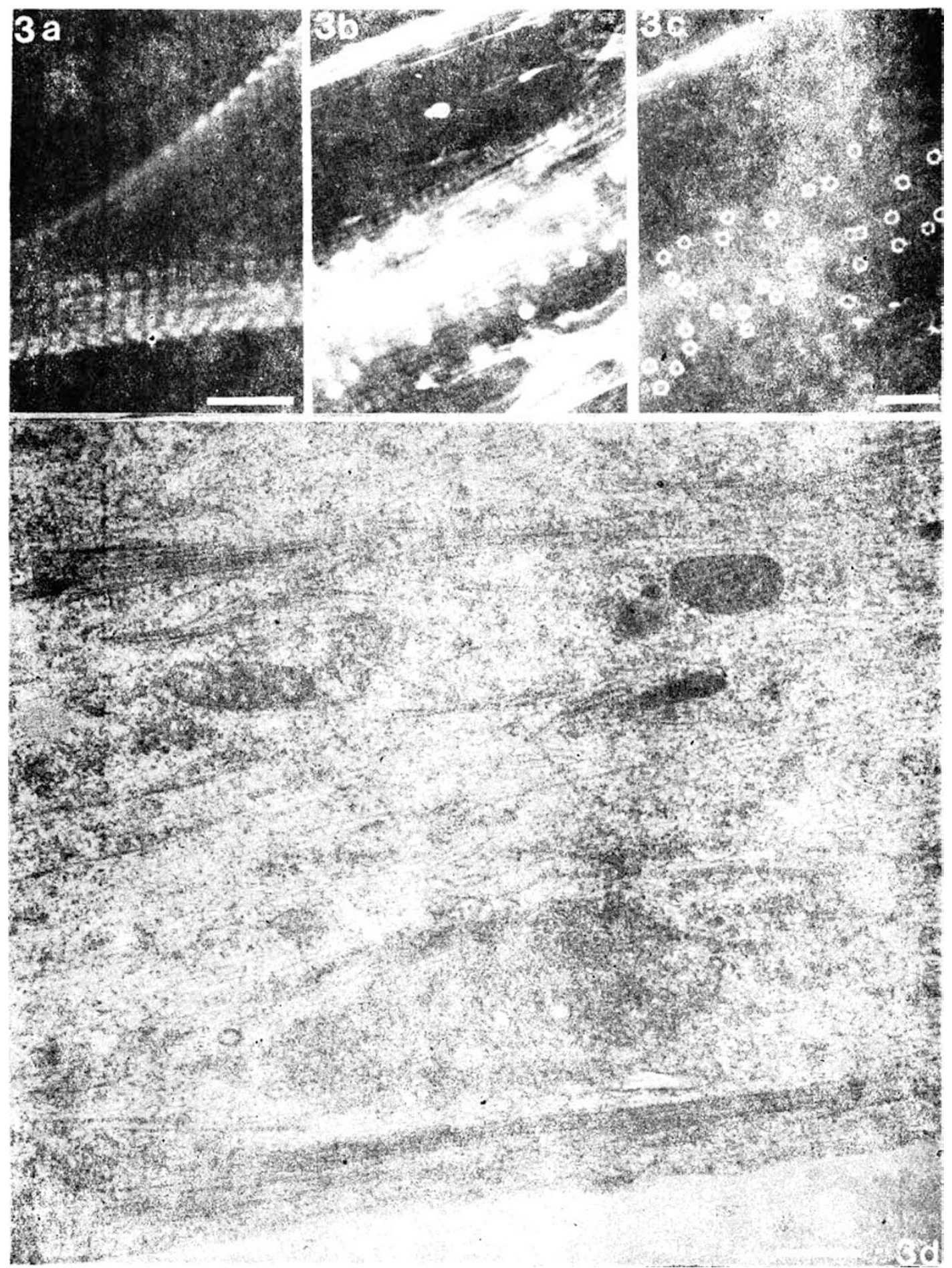

Fig. 3 (a) a-actin immunofluorescent staining of myotube of a 4-day culture of chicken embryonic myoblasts, showing I-band striations. (b) and (c) are from same field of TPA-treated 4-day myotubes. Rho-phalloidin staining in (b) showing actin bodies. Vinculin immunofluorescence in (C) showing colocalization of vinculin rims with actin bodies. (d) EM micrograph showing ultrastructures of the TPA-induced CAB (arrow). bar: $10 \mu \mathrm{m}$ (a, b, c) $1 \mu \mathrm{m}(\mathrm{d})$. 